\title{
Optimizing parametrial aperture design utilizing HDR brachytherapy isodose distribution
}

\author{
Katherine L. Chapman', Nitin Ohri², Timothy N. Showalter ${ }^{3}$, Laura A. Doyle \\ IDepartment of Radiation Oncology, Jefferson Medical College of Thomas Jefferson University, Kimmel Cancer Center, Philadelphia, PA. \\ 2Department of Radiation Oncology, Montefiore Medical Center, Albert Einstein College of Medicine, Bronx, NY, ${ }^{3}$ Department of Radiation \\ Oncology, University of Virginia, Charlottesville, VA, USA
}

\begin{abstract}
Treatment of cervical cancer includes combination of external beam radiation therapy (EBRT) and brachytherapy (BRT). Traditionally, coronal images displaying dose distribution from a ring and tandem (R\&T) implant aid in construction of parametrial boost fields. This research aimed to evaluate a method of shaping parametrial fields utilizing contours created from the high-dose-rate (HDR) BRT dose distribution.

Eleven patients receiving HDR-BRT via R\&T were identified. The BRT and EBRT CT scans were sent to FocalSim $(\mathrm{v} 4.62)^{\circledR}$ and fused based on bony anatomy. The contour of the HDR isodose line was transferred to the EBRT scan. The EBRT scan was sent to CMS-XIO $(\mathrm{v} 4.62)^{\circledR}$ for planning. This process provides an automated, potentially more accurate method of matching the medial parametrial border to the HDR dose distribution. This allows for a 3D-view of dose from HDR-BRT for clinical decision-making, utilizes a paperless process and saves time over the traditional technique.

Key words: brachytherapy, cervical cancer, combined modality.

\section{Purpose}

Radiation therapy has been established as an effective component of definitive treatment of patients with invasive cervical cancer. Current guidelines recommend a cumulative EQD2, equivalent dose in 2 Gy fractionation of 75-85 Gy to the parametrial tissues [1-3]. This is typically delivered with a combination of external beam radiation therapy (EBRT) and brachytherapy (BRT). Combination therapy allows for delivery of increased dose to tumor while sparing surrounding normal tissue. A portion of the EBRT dose is often delivered as a parametrial/pelvic sidewall boost that spares midline structures. A variety of techniques have been used to design the parametrial boost fields and are described in the literature [4-6]. It is imperative that each phase of radiotherapy (EBRT, brachytherapy, and parametrial boost) is designed and implemented properly as increased local control and survival are expected when the entire treatment course delivers the appropriate dose and is completed within eight weeks $[1,7,8]$.

At our institution, cervical cancer patients are typically treated with a traditional 4-field, conformal pelvis technique, a parametrial boost plan with parallel-opposed AP and PA fields, and high dose rate (HDR) intracavitary brachytherapy. Our practice to date has been to manually design the parametrial boost fields, so that they are situated immediately lateral to the $50 \%$ isodose lines of the HDR brachy- therapy plans. In this report, we explore a simple, elegant, image-based method of designing the parametrial boost fields by utilizing contours created from HDR BRT dose distribution using computer-based planning.

\section{Material and methods}

\section{Patient selection}

Eleven consecutive patients with locally advanced cervical carcinoma (stage IIB - 10, and IIIB - 1) treated between August 2009 and October 2011 were identified. Nine of the eleven patients had bilateral parametrium involvement. All patients were treated at a single institution for all components of radiation therapy. Fractionation schemes include initial external beam to the whole pelvis for $45 \mathrm{~Gy}$ in 25 fractions, total brachytherapy doses ranging from 29-35 Gy in 5 fractions and parametrial boost doses of 5.4-14.4 Gy in 3-8 fractions (1.8 Gy/fraction).

\section{Treatment planning objectives}

All patients were treated with external beam radiation therapy (EBRT) for whole pelvis irradiation followed by intracavitary brachytherapy and a boost to the parametrium. CT image sets were obtained for EBRT and BRT planning using GE-LightSpeed 16-slice scanner ${ }^{\circledR}$ (GE Healthcare, Wau- 
kesha, WI, USA). EBRT planning was performed using CMS $\mathrm{XIO} 4.62{ }^{\circledR}$ (Nucletron, an Elekta company, Elekta AB, Stockholm, Sweden). Traditional four-field, conformal RT technique was used for the initial pelvis fields to a total dose of 45 Gy using standard dose/fractionation scheme of $1.8 \mathrm{~Gy}$ daily fractions over the course of 5 weeks. One patient received two larger fractions of $3 \mathrm{~Gy}$ at the onset of therapy to control bleeding. Towards the end of the EBRT course, all patients received HDR brachytherapy on non-EBRT days with an effort to keep total treatment time within eight weeks. All patients were treated with a CT/MR compatible intrauterine ring, tandem applicator of varying size, angulations and Nucletron V2 afterloader ${ }^{\circledR}$ (Nucletron, an Elekta company, Elekta AB, Stockholm, Sweden). The brachytherapy dose was prescribed to point $\mathrm{A}, 2 \mathrm{~cm}$ superior and $2 \mathrm{~cm}$ lateral to the cervical os, as defined by the superior surface of the ring, detailed in the American Brachytherapy Society consensus guidelines [3] . All brachytherapy planning was performed using Oncentra MasterPlan $3.2^{\circledR}$ (Nucletron, an Elekta company, Elekta AB, Stockholm, Sweden). All parametrial boost plans were achieved with aperture-based parallel-opposed anterior and posterior fields. Planning of the boost fields was performed immediately following the first fraction of HDR brachytherapy to ensure no gap in treatment.

\section{Aperture design of boost fields}

Current procedure requires a single coronal slice, displaying the isodose lines resulting from patient's first HDR BRT treatment printing. The physician then designs the boost fields on EBRT planning CT by visually matching medial field borders to the $50 \%$ isodose line displayed on paper, and evaluates dose to the target volume and critical structures. For this study, the CT image-set and three-dimensional dose distribution from each patient's first ring and tandem HDR brachytherapy fraction was exported to MIM Vista 5.1 (Fig. 1). Each case was loaded in MIM Vista and the $50 \%$ isodose surface (IDS) was converted into a contour. The CT image-set and the structure set containing the $50 \%$ IDS contour was transferred to FocalSim $4.62{ }^{\circledR}$ (Elekta company, Elekta AB, Stockholm, Sweden) where it was fused to the EBRT planning CT image-set (Fig. 2). Due to soft tissue deformation caused by the intrauterine applicator, the fusion was assessed solely on bony anatomy. Once fused, the $50 \%$ IDS contour was copied to the EBRT planning CT image-set. In CMS XIO ${ }^{\circledR}$ (Elekta company, Elekta $\mathrm{AB}$, Stockholm, Sweden), the original parametrial boost plan based on single coronal slice showing isodose lines was loaded (Table 1). The medial border of the existing anterior field edge was adjusted to conform to the $50 \%$ IDS contour (Fig. 3). This process was repeated for the posterior field edge. Dose normalization was matched to the original plan.

\section{Plan assessment}

Field size as indicated by blocked equivalent square was analyzed for initial and edited plan. Volume of irradiated tissue was assessed in MIM by creating contours from the $85 \%, 90 \%$, and $95 \%$ isodose lines using the process described above. The volume encompassed by these lines was used for comparison.

\section{Results}

Nine of eleven patients were clinically deemed to require bilateral boost fields to left and right parametrial nodes.

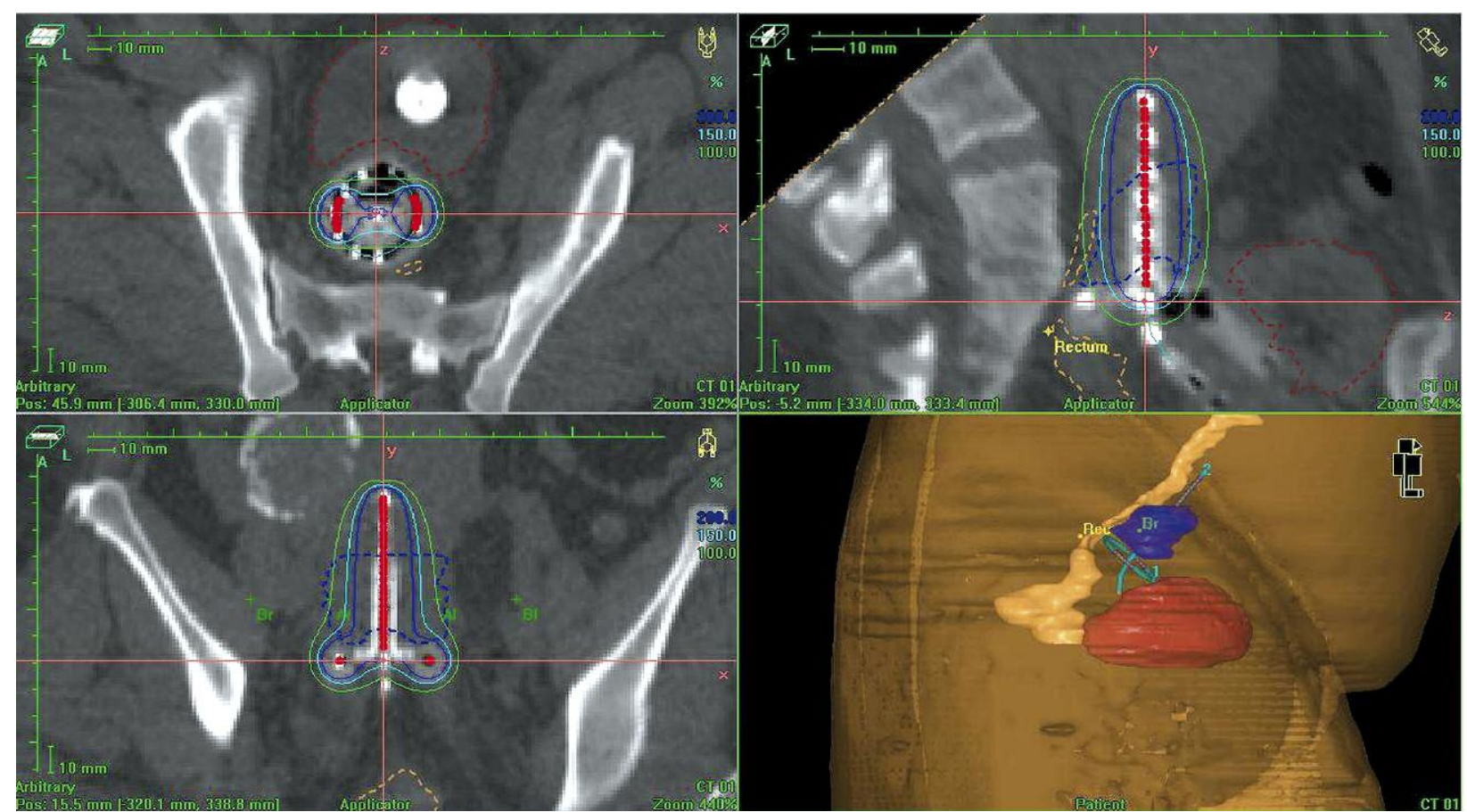

Fig. 1. Dose distribution of typical ring and tandem implant shown in axial, sagittal and coronal planes. Plan generated in brachytherapy module of Oncentra MasterPlan and dose normalized to point A 

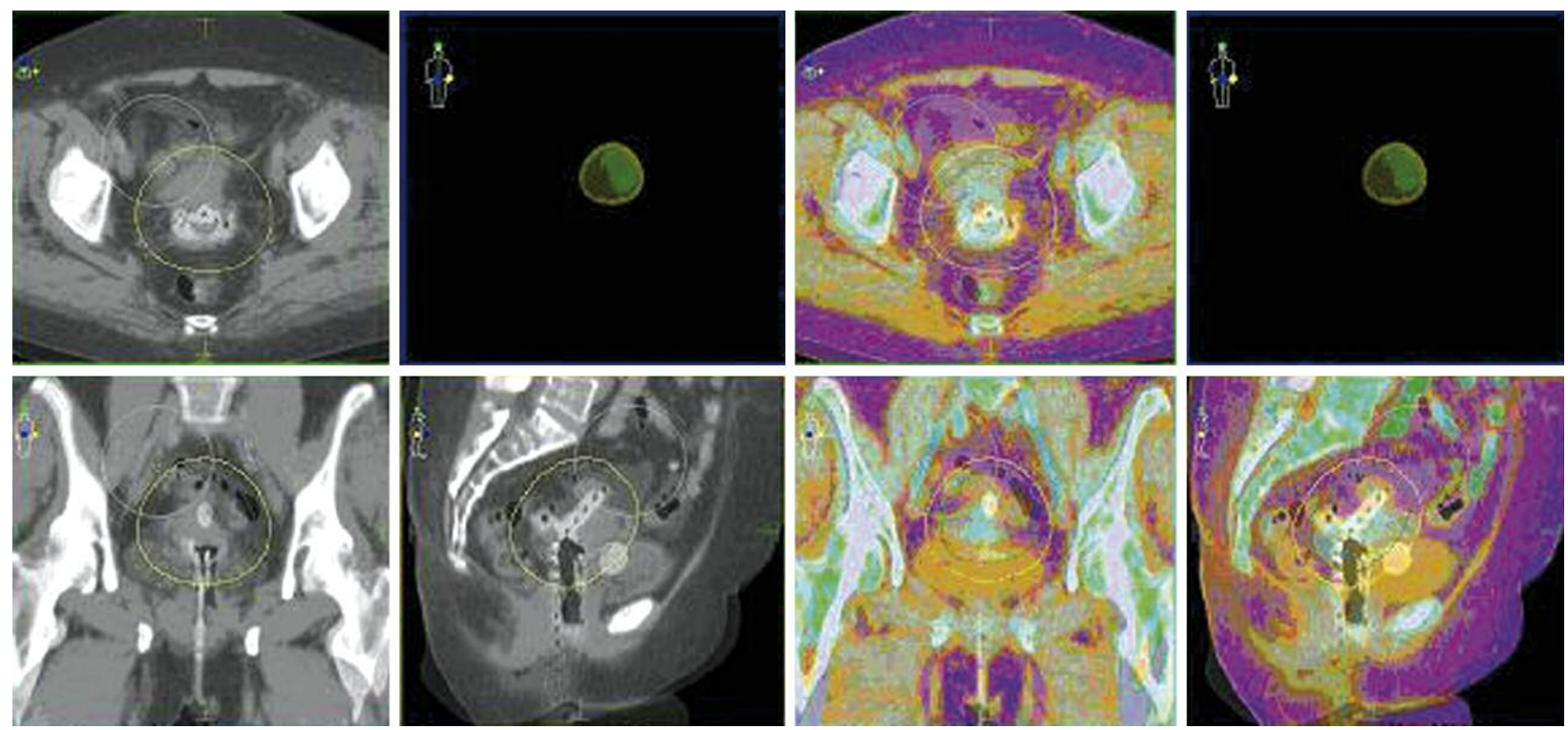

Fig. 2. Axial slices showing fusion of external beam planning CT image-set and brachytherapy planning CT image-set in Focal$\mathrm{Sim}$. The $30 \%$ and $50 \%$ isodose surfaces are shown on brachytherapy planning CT

Table 1. Field size as indicated by blocked equivalent square in CMS XIO

\begin{tabular}{|c|c|c|c|c|c|c|c|c|c|c|c|c|}
\hline & \multicolumn{12}{|c|}{ Blocked Equivalent Square (cm) } \\
\hline & \multicolumn{4}{|c|}{ Initial Boost } & \multicolumn{4}{|c|}{ Replanned Boost } & \multicolumn{4}{|c|}{ Difference } \\
\hline & \multicolumn{2}{|c|}{ Right } & \multicolumn{2}{|c|}{ Left } & \multicolumn{2}{|c|}{ Right } & \multicolumn{2}{|c|}{ Left } & \multicolumn{2}{|c|}{ Right } & \multicolumn{2}{|c|}{ Left } \\
\hline & AP & $\mathrm{PA}$ & $A P$ & PA & AP & $\mathrm{PA}$ & AP & PA & $A P$ & PA & $\mathrm{AP}$ & PA \\
\hline Mean & 4.54 & 4.61 & 4.51 & 4.50 & 3.94 & 4.10 & 3.90 & 3.96 & 0.60 & 0.51 & 0.61 & 0.54 \\
\hline Standard deviation & 0.97 & 0.94 & 0.53 & 0.59 & 0.94 & 0.96 & 0.70 & 0.61 & 0.78 & 0.75 & 0.63 & 0.56 \\
\hline
\end{tabular}
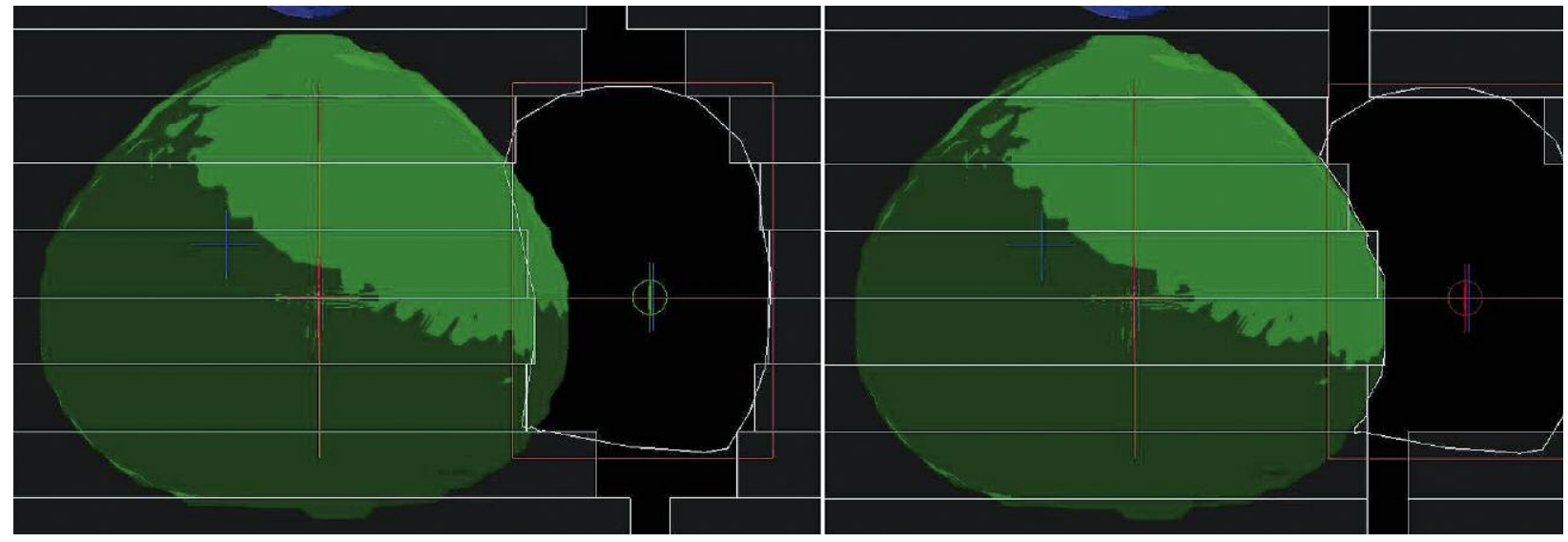

Fig. 3. Left image shows original MLC aperture based on hardcopy images of coronal slices from HDR plan. Right image shows the same MLC aperture with medial border conformed to the contour of the $50 \%$ isodose surface

Thus, 20 parametrial regions were treated, each with two fields (AP and PA). Of 40 fields analyzed, 7 increased and 33 decreased in size when this novel technique was used. Initial field sizes ranged from $2.94 \mathrm{~cm}$ to $6.36 \mathrm{~cm}$. Replanned field sizes ranged from $2.61 \mathrm{~cm}$ to $5.98 \mathrm{~cm}$. The mean change in field size was a decrease of $0.56 \mathrm{~cm}$ (range: -0.17 to $2.05 \mathrm{~cm}$ ). The volumes encompassed by the $95 \%, 90 \%$, and $80 \%$ isodose surfaces of the parametrial boost fields decreased by an average of $23.1 \mathrm{cc}, 48.5 \mathrm{cc}$, and $71.1 \mathrm{cc}$, re- spectively (Table 2). These changes were all statistically significant $(p<0.05)$ using paired Student's $t$-test.

\section{Discussion}

This work presents results of a novel technique for shaping parametrial fields based on contours formed from HDR BRT dose distributions. For a majority of patients, designing parametrial fields based on the contour derived from 
the $50 \%$ IDS resulted in a decrease in field size as indicated by the blocked equivalent square and volume of irradiated tissue. The goal of parametrial field design is to supplement central dose distribution from HDR brachytherapy to extend laterally into parametrial nodal regions, providing a total dose of 50-55 Gy to this area treated with initial whole pelvis EBRT. Thus, the field design is dependent upon HDR dose distribution. Although the classic pear shaped distribution created by the ring and tandem applicator is fairly consistent, there are slight changes in shape and size due to different combinations of applicator lengths and angles. Traditional methods rely on the clinician to qualitatively match the border of the HDR dose distribution. In the era of 3D brachytherapy dose calculations and ease of DICOM transfer, the method proposed in this work allows for quantitative assessment of field design and improves consistency in the process of field design. Although quantitative time analysis for the two techniques was not conducted, the proposed technique can be completed in less than 15 minutes. Optimal design of parametrial fields should be established to avoid possible over- or under-dosing of the nodal regions. It has been shown that high dose to the parametrium can result in an increased likelihood of developing radiation proctitis [9] and enterocolitis [10]. As stated, this technique resulted in a reduction of field size in $82.5 \%$ of parametrial fields indicating a likely decrease in possibility of regions of increased dose. For treatment using techniques other than traditional anterior/posterior fields, contours of various isodose surfaces could serve as avoidance structures. This method could further reduce overdosing pelvic sidewall regions. Future research should be done to include total composite dose assessment from all treatment modalities, however this task is complicated by the need for deformable registration and equivalent dose considerations.

There are several limitations to this study. In our clinical practice, a new plan is generated for each R\&T implant. In this study, however, the isodose contour used to shape the medial border of the parametrial field represents dose distribution from a single fraction. Differences in dose distributions between fractions were not considered. In addition, our sample size of eleven patients was limited. More subtle differences between the fields generated with each technique may be identified if more patients are studied.

This study did not address many advances in brachytherapy of the cervix, including use of MR imaging in contouring and planning or prescription to a high risk clinical target volume as recommended by the American Brachytherapy Society [3]. There are a number of manuscripts describing the advantages of MRI in delineating the high risk clinical target volume HR-CTV and critical organs at risk described by the GEC-ESTRO working group [11]. There are also advantages to MRI in the pre-planning setting [12], and to utilize volume based planning to decrease the variability seen when prescribing to point $A$, including the changes in the definition of Point A over time [13]. Although MR guidance and target volume delineation is ideal, a recent study found that approximately $77 \%$ of institutions in the United States still prescribe to Point A [14]. This technique we describe may be utilized regardless of brachytherapy planning technique as a way to better design parametrial fields around the brachytherapy dose distribution.
Table 2. Change in volume of tissue encompassed by $95 \%, 90 \%$, and $80 \%$ isodose surfaces after replan

\begin{tabular}{|c|c|c|c|}
\hline & & Mean & Standard deviation \\
\hline \multicolumn{4}{|l|}{$95 \%$ IDL (cc) } \\
\hline \multirow[t]{2}{*}{ Initial Boost } & Right & 64.10 & 40.05 \\
\hline & Left & 65.78 & 42.14 \\
\hline \multirow[t]{2}{*}{ Replanned Boost } & Right & 50.10 & 48.18 \\
\hline & Left & 35.28 & 22.53 \\
\hline \multirow[t]{2}{*}{ Difference } & Right & 14.00 & 24.09 \\
\hline & Left & 30.51 & 30.51 \\
\hline \multicolumn{4}{|l|}{$90 \%$ IDL (cc) } \\
\hline \multirow[t]{2}{*}{ Initial Boost } & Right & 164.46 & 96.58 \\
\hline & Left & 159.64 & 76.13 \\
\hline \multirow[t]{2}{*}{ Replanned Boost } & Right & 124.30 & 107.30 \\
\hline & Left & 104.23 & 50.47 \\
\hline \multirow[t]{2}{*}{ Difference } & Right & 40.16 & 62.96 \\
\hline & Left & 55.40 & 48.02 \\
\hline \multicolumn{4}{|l|}{$80 \%$ IDL (cc) } \\
\hline \multirow[t]{2}{*}{ Initial Boost } & Right & 340.35 & 138.48 \\
\hline & Left & 305.78 & 83.10 \\
\hline \multirow[t]{2}{*}{ Replanned Boost } & Right & 263.02 & 138.23 \\
\hline & Left & 238.67 & 89.36 \\
\hline \multirow[t]{2}{*}{ Difference } & Right & 77.33 & 108.84 \\
\hline & Left & 67.11 & 59.34 \\
\hline
\end{tabular}

\section{Conclusions}

The size and shape of the parametrial boost fields changed relatively consistently, but not dramatically, by conforming the medial field edge to the contour of the $50 \%$ isodose line from the HDR plan. While the clinical implications of these changes are difficult to predict, this technique may lead to more efficient and reliable treatment planning. This process provides a simple, automated, potentially more accurate, method of matching the medial parametrial border to the HDR dose distribution, provides a $3 \mathrm{D}$ view of the dose from HDR BRT for clinical decision making, utilizes a paperless process and may save time over the traditional technique. However, accuracy is highly dependent on image fusion of HDR and EBRT CTs, which remains difficult due to differences in patient setup and tissue deformation. In all cases, the fusion process requires additional physician review and approval.

\section{References}

1. Vishwanathan A, Beriwal S, De Los Santos J et al. American Brachytherapy Society consensus guidelines for locally advanced carcinoma of the cervix. Part II: High-dose-rate brachytherapy. Brachytherapy 2012; 11: 47-52.

2. Toita T, Kakinohana Y, Ogawa K et al. Combination external beam radiotherapy and high-dose-rate intracavitary brachytherapy for uterine cervical cancer: analysis of dose and fractionation schedule. Int J Radiat Oncol Biol Phys 2003; 56: 1344-1353.

3. Viswanathan AN, Thomadsen B; American Brachytherapy Society Cervical Cancer Recommendations Committee; Ameri- 
can Brachytherapy Society. American Brachytherapy Society consensus guidelines for locally advanced carcinoma of the cervix. Part I: General principles. Brachytherapy 2012; 11: 33-46.

4. Good J, Lalondrelle S, Blake P. Point: Parametrial irradiation in locally advanced cervix cancer can be achieved effectively with a variety of external beam techniques. Brachytherapy 2012; 11: 77-79.

5. Stock R, Chen A, Flickinger J et al. Node-positive cervical cancer: impact of pelvic irradiation and patterns of failure. Int J Radiat Oncol Biol Phys 1994; 31: 31-36.

6. Fenkell L, Assenholt M, Nielsen SK et al. Parametrial boost using midline shielding results in an unpredictable dose to tumor and organs at risk in combined external beam radiotherapy and brachytherapy for locally advanced cervical cancer. Int J Radiat Oncol Biol Phys 2011; 79: 1572-1579.

7. Chen SW, Liang JA, Yang SN et al. The adverse effect of treatment prolongation in cervical cancer by high-dose-rate intracavitary brachytherapy. Radiother Oncol 2003; 67: 69-76.

8. Lanciano RM, Martz K, Coia LR et al. Tumor and treatment factors improving outcome in stage III-B cervix cancer. Int J Radiat Oncol Biol Phys 1991; 20: 95-100.

9. Lin $\mathrm{H}, \mathrm{Hsu} \mathrm{HC}$, Wang $\mathrm{CJ}$ et al. High external parametrial dose can increase the probability of radiation proctitis in patients with uterine cervix cancer. Gynecol Oncol 2000; 79: 406-410.

10. Huang EY, Wang CJ, Hsu HC et al. Dosimetric factors predicting severe radiation-induced bowel complications in patients with cervical cancer: combined effect of external parametiral dose and cumulative rectal dose. Gynecol Oncol 2004; 95: 101-108.

11. Haie-Meder C, Pötter R, Van Limbergen E et al. Recommendations from gynaecological (GYN) GEC-ESTRO working group (I): Concepts and terms in 3D image based 3D treatment planning in cervix cancer brachytherapy with emphasis on MRI assessment of GTV and CTV. Radiother Oncol 2005; 74: 235-245.

12. Petric P, Hudej R, Music M. MRI assisted cervix cancer brachytherapy pre-planning, based on insertion of the applicator in para-cervical anaesthesia: preliminary results of a prospective study. J Contemp Brachytherapy 2009; 1: 163-169.

13. Anderson J, Huang Y, Kim Y. Dosimetric impact of point A definition on high-dose-rate brachytherapy for cervical cancer: evaluations on conventional point A and MRI-guided, conformal plans. J Contemp Brachytherapy 2012; 4: 241-246.

14. Viswanathan AN, Erickson BA. Three-dimensional imaging in gynecologic brachytherapy: a survey of the American Brachytherapy Society. Int J Radiat Oncol Biol Phys 2010; 76: 104-109. 